\title{
Riesgos dietéticos y alimentos ultraprocesados
}

\section{Dietary risks and ultra-processed foods}

Hipócrates dijo "que el alimento sea tu mejor medicina", tratando de hacernos recordar la importancia de una alimentación adecuada para poder tener una vida saludable; sin embargo, este consejo parece no ser considerado en la actualidad por cuanto hay una crisis nutricional, tal como nos lo recuerda un reciente artículo publicado en la revista Lancet (1). Los autores de este articulo afirman que "la malnutrición en todas sus formas, incluyendo la obesidad...es la causa principal de la pobre salud global". El problema nutricional debe ser visto desde todos sus ángulos, siendo necesario establecer recomendaciones optimas de ingesta calórica buscando dietas saludables que impacten la salud global y evitando dietas que conlleven un riesgo para la salud.

Hablar de mala nutrición implica reconocer a 3 grupos de personas: obesos, desnutridos y aquellos con "riesgos dietéticos" (dietas bajas en granos enteros, frutas, vegetales, nueces, y semillas y en su lugar dietas con alto contenido de sodio, carnes rojas y bebidas azucaradas). En este último grupo están también los alimentos ultraprocesados.

A nivel global, el número de personas expuesta a "riesgos dietéticos" sumado a aquellos con obesidad está por encima del número de hipertensos, fumadores y personas con glucosa alta en ayunas. Este aumento si bien es más marcado en poblaciones de alto o medio alto índice de desarrollo social, se observa también en el grupo de países de medio y medio bajo índice de desarrollo social (2). Lo preocupante es que no es solo un problema de población adulta, sino que niños y adolescentes también son víctimas de esta epidemia (3). Se estima que en el 2015 cerca de 268 millones de niños y adolescentes en 200 países tendrán sobrepeso, 124 millones obesidad y tres cuartas partes de la morbi-mortalidad asociada a esto va a ocurrir en países de ingresos medios bajos.

En este número de la Revista Médica Herediana se presenta un estudio descriptivo, observacional donde se estudia la frecuencia de consumo de alimentos ultra procesados y su relación con parámetros antropométricos en alumnos entre 9 y 17 años de un colegio del Agustino en Lima. A pesar de sus limitaciones el estudio nos muestra como el consumo de alimentos ultraprocesados (evaluado a través de una encuesta) se puede asociar a una mayor frecuencia de sobrepeso y obesidad en niños de primaria. Los autores establecen también como en estudiantes de primaria otros parámetros antropométricos como IMC, IMC/Edad y perímetro abdominal guardan relación directa con la ingesta de alimentos ultraprocesados; no observándose esto en estudiantes de secundaria. A pesar de que el consumo diario de alimentos ultraprocesados no fue lo más frecuente (solo se dio en $42 \%$ de los estudiantes de primaria y $39.5 \%$ de los estudiantes de secundaria), $51(46,4 \%)$ de los estudiantes de primaria y $58(44,5 \%)$ de los estudiantes de secundaria presentaban sobrepeso u obesidad. Pese a ser una muestra pequeña y poco extrapolable nos llama la atención este porcentaje, por cuanto se trata de un colegio en una zona de Lima donde el $60 \%$ de las familias tienen un estrato socioeconómico medio bajo o bajo (4).

En nuestro país se han publicado estudios evaluando la prevalencia de sobrepeso y obesidad en niños. La Dra. Preston, realizó un análisis secundario de los datos de un estudio longitudinal de niños en pobreza (5). En este estudio se siguió una cohorte de niños entre 7 y 8 años en el año 2009. La prevalencia de sobrepeso fue de $19,2 \%$ y de obesidad 8,6\%, observándose esto en los niños provenientes de un estrato socioeconómico más alto. En otro estudio que analizó los datos del Sistema de Información del Estado Nutricional, se encontró que entre los años 2010 y 2015 la prevalencia de sobrepeso y obesidad a nivel nacional fue de $6,2 \%$ a $6,8 \%$ y de $1,5 \%$ a $2,7 \%$ respectivamente. Se observó una mayor frecuencia de sobrepeso y obesidad en las zonas de costa, especialmente en Lima, Callao, Ica, Moquegua y Tacna (6). Villena J. (7), en una revisión sobre sobrepeso y obesidad en el Perú, al describir población pediátrica y adolescentes analizando los datos de la Encuesta Nacional de Hogares (ENAHO) encuentra que en menores de 5 años el 8,2\% presenta exceso de peso, y entre los 5 a 19 años, 24,4\% presentan exceso de peso. 
El consumo de alimentos ultraprocesados es frecuente y se ha encontrado que conlleva un mayor riesgo de mortalidad en adultos de edad mediana como se ha descrito recientemente en una cohorte francesa de más de 44,000 sujetos en quienes se evaluó hábitos nutricionales (8). Si bien el estudio fue en población adulta, el consumo de alimentos ultraprocesados fue mayor en sujetos de menor ingreso socioeconómico, menor nivel educativo y menor edad.

Algunos ejemplos de productos típicos ultraprocesados son: bebidas carbonatadas; aperitivos envasados dulces o salados; helados, chocolates, dulces (confitería); panes y pasteles; margarinas y productos para untar; galletas y tortas, "cereales" y barras de "energía"; bebidas energizantes; bebidas de leche, yogures y bebidas de frutas; bebidas de cacao; extractos de carne y pollo y salsas instantáneas; muchos productos listos para calentar. También están considerados pasteles preparados y platos de pasta y pizza; nuggets de aves de corral y pescado y salchichas, hamburguesas, hot dogs y otros productos cárnicos reconstituidos, y en polvo y empacadas; sopas instantáneas, fideos y postres $(9,10)$. Es pues importante educar a la comunidad en los riesgos asociados al consumo de alimentos ultraprocesados y cuales son estos alimentos con un claro "riesgo dietético".

Hipócrates tenía razón cuando nos recuerda en su aforismo 69 que "Los obesos están más expuestos a muerte" (11). La mayoría de los productos que se expenden en los supermercados y bodegas son ultraprocesados y el ritmo agitado de la vida actual hace que mucha gente joven opte por alimentos de "comida rápida". La calidad nutricional de estos alimentos es muy mala y no se puede negar que son extremadamente sabrosos y casi adictivos (10). Como profesionales de la salud es esencial tomar conciencia de que el problema nutricional en el mundo actual ya no es tanto la desnutrición calórico-proteica, sino también una nutrición inadecuada que favorece el desarrollo de sobrepeso y obesidad, lo cual va a impactar negativamente en la salud. No solo los excesos dietéticos son dañinos para nuestra salud, sino la calidad de los alimentos que ingerimos, y en los alimentos ultraprocesados hay un claro riesgo de mortalidad.

Eduquemos a nuestra comunidad, para cambiar los hábitos dietéticos negativos. El estudio de Lozano VM. (12), nos recuerda que las dietas ultraprocesadas son una realidad incluso en poblaciones que no son de un alto nivel socioeconómico, aun cuando la mayoría de los niños encuestados en su estudio hayan tenido un consumo menor de alimentos ultraprocesados. El uso de octógonos en los alimentos ultraprocesados sin duda va a ayudar a tomar una mayor conciencia de la importancia de una dieta saludable.

Luis Manuel Valdez ${ }^{1}$

\section{REFERENCIAS BIBLIOGRÁFICAS}

1. Swinburn BA, Kraak VI, Allender S, Atkins VJ, Baker PI et al. The Global Syndemic of Obesity, Undernutrition, and Climate Change: The Lancet Commission report. Lancet 2019; 393:791-846

2. NCD Risk Factor Collaboration (NCD-RisC). Trends in adult body-mass index in 200 countries from 1975 to 2014: a pooled analysis of 1698 population-based measurement studies with $19 \cdot 2$ million participants. Lancet. 2016; 387: 1377-1396

3. Afshin A, Forouzanfar MH, Reitsma MB, et al, and the GBD 2015 Obesity Collaborators. Health effects of overweight and obesity in 195 countries over 25 years. N Engl J Med 2017; 377: 13-27.
4. INEI. Planos Estratificados de Lima Metropolitana 2016. Según ingreso per capita del Hogar. Según grupos de Pobreza Metropolitana. (citado el 2 de junio del 2019). Disponible en: https://www.inei.gob. pe/media/MenuRecursivo/publicaciones_digitales/ Est/Lib1403/libro.pdf

5. Preston EC, Ariana P, Penny ME, Frost M, Plugge E. Prevalence of childhood overweight and obesity and associated factors in Peru. Rev Panam Salud Publica. 2015;38(6):472-8

6. Smith Torres-Roman J, Urrunaga-Pastor D, Avilez JL, Helguero-Santin LM, Malaga G. Geographic differences in overweight and obesity prevalence in Peruvian children, 2010-2015. BMC Public Health. 2018; 18: 353-61. 
7. Villena Chavez JE. Prevalencia de Sobrepeso y Obesidad en el Peru. Revista Peruana de Ginecología y Obstetricia 2017; 63: 593-59.

8. Schnabel L, Kesse-Guyot E, Allès B, et al. Association between Ultraprocessed Food Consumption and Risk of Mortality among Middle-aged Adults in France. JAMA Intern Med. 2019

9. Monteiro CA, Cannon G, Levy RB et al. NOVA. The star shines bright. [Food classification. Public health]. World Nutrition 2016; 7: 28-38
10. Alimentos y bebidas ultraprocesados en América Latina: tendencias, efecto sobre la obesidad e implicaciones para las políticas públicas. Washington, DC: OPS, 2015.

11. Hipócrates. Aforismos y sentencias. (citado el 3 de junio del 2019). Disponible en https:/www. ellibrototal.com/ltotal/?t=1\&d=6857

12. Lozano VM, Hermoza-Moquillaza RV, ArellanoSacramento C; Hermoza-Moquillaza VH. Relación entre ingesta de alimentos ultra procesados y los parámetros antropométricos en escolares. Rev Med Hered. 2019; 30:68-75. 